WPSReview International on Sustainable Housing and Urban Renewal

(RI-SHUR)

\title{
ANÁLISIS DE LAS INSTALACIONES DE ALUMBRADO PÚBLICO EN ESPAÑA A TRAVÉS DE CONCURSOS PÚBLICOS Y TENDENCIAS DE RENOVACIÓN Y MEJORA EN BASE A LA EFICIENCIA ENERGÉTICA
}

\author{
Rubén Pérez Maldonado \\ Alfonso Gago Calderón \\ Universidad de Málaga
}

Artículo Recibido: 05/10/2016

Artículo Aceptado: 14/06/2017

\section{Resumen}

El aumento de la conciencia medioambiental y la necesidad de optimizar los recursos públicos de los municipios están impulsando en la actualidad un proceso generalizado de mejorar de las instalaciones de alumbrado público en un gran número de núcleos urbanos de todo el país. Esta tendencia coincide y encuentra su motivación y justificación esencialmente con el auge de la comercialización de equipos de iluminación basados en tecnología LED.

Las actuaciones de las instalaciones, a nivel público, se están planteando y ejecutando mediante la convocatoria paulatina de concursos públicos. En este trabajo se ha recopilado información de múltiples "Pliegos de Cláusulas Administrativas" y "Pliegos de Preinscripciones Técnicas", algunos con proyectos o auditorias de ahorro energéticos externos anexos, publicados entre 2014 y 2016 de múltiples localizaciones geográficas, tamaños de población y objetivos a alcanzar, con los que hemos diseñado un boceto del estado general en el que se encontraban este tipo de instalaciones con tecnologías de iluminación convencionales y como se están actualizando para cumplir con: 
- Los nuevos reglamentos vigentes, tanto de eficiencia energética vigente como, si existen para su Comunidad Autónoma, de protección de cielo nocturno contra la contaminación lumínica

- Establecer nuevos criterios de seguridad y garantía de calidad en este tipo de instalaciones como los establecidos por el Comité Español de lluminación (en adelante CEI)

- Conseguir los máximos ahorros energéticos y económicos posibles.

Palabras Clave: Alumbrado Público; lluminación LED; Concursos Públicos

\section{Abstract}

Increased environmental awareness and the need to optimize public resources of municipalities are driving today a generalized process of improving street lighting facilities in a number of towns across the country. This trend coincides and finds its motivation and justification essentially with the rise of marketing of lighting equipment based on LED technology.

The performances of facilities at the public level, are considering running through the gradual and call for public tenders. This paper has gathered information from multiple Sheets of Administrative Clauses and tender documents Pre-registration techniques, some projects or audits of external energy savings annexes, published between 2014 and 2016 multiple geographic locations, population sizes and objectives to be achieved, with the we have designed a sketch of the general state in which such facilities were conventional lighting technologies as being upgraded to meet:

- The new current regulations, both current as energy efficiency, if they exist for their autonomous region, night sky protection against light pollution.

- $\quad$ Establish new criteria for safety and quality assurance facilities such as those established by the Spanish Lighting Committee (CEI) and the Institute for Diversification and Saving of Energy (IDAE), Ministry of Industry Government of Spain.

- $\quad$ Getting the maximum possible energy and economic savings. 


\section{1.- Introducción}

La iluminación nocturna, a través de su evolución y desarrollo, ha contribuido a mejorar notablemente tanto la seguridad ciudadana como la seguridad viaria y ha permitido el desarrollo de actividades comerciales, productivas, deportivas y lúdicas a determinadas horas de la noche.

La eficiencia energética en las instalaciones de alumbrado público es un aspecto de mucha actualidad en el marco presente a nivel mundial. El desarrollo de nuevas tecnologías de iluminación junto con la elevada antigüedad media de este tipo de instalaciones de España, la creciente concienciación sobre eficiencia energética, productividad y cuidado medioambiental y parámetros de mejora de las cuentas de resultados económicos de los estamentos públicos (ayuntamientos, diputaciones y ministerios) están apoyando esta tendencia.

En este trabajo realizamos un análisis detallado de un conjunto significativo de concursos públicos relacionados con actuaciones sobre instalaciones de alumbrado público publicados entre 2014 y 2016, mezclando múltiples localizaciones geográficas, tamaños de población y objetivos a alcanzar, con los que hemos diseñado un boceto del estado en el que se encuentran este tipo de instalaciones con tecnologías de iluminación convencionales y cómo se están actualizando para cumplir con:

- Los nuevos reglamentos, tanto de eficiencia energética vigente como, si existen para su comunidad autónoma, de protección de cielo nocturno contra la contaminación lumínica

- $\quad$ Establecer nuevos criterios de seguridad y garantía de calidad como los establecidos por el Comité Español de lluminación (en adelante CEI) y el Instituto para la Diversificación y Ahorro de la Energía (en adelante IDAE) del Ministerio de Industria del gobierno de España

- Conseguir los máximos ahorros energéticos y económicos posibles.

Hemos recopilado información de sus "Pliegos de Cláusulas Administrativas" y "Pliegos de Preinscripciones Técnicas" de concursos tanto de suministro como de obras de instalación o contratación de Empresas de Servicios Energéticos 
para actualizar y mantener estas instalaciones. Algunos casos se acompañan con proyectos o auditorias de ahorro energéticos externos anexos que recogen detalladamente el estado de partida preciso de la instalación que se quiere remodelar.

Estos documentos son una radiografía detallada de un sector tecnológico de alta relevancia en cualquier tipo de país industrializado donde la iluminación es un indicador muy significativo del grado de desarrollo de una nación y un aspecto muy valorado por sus poblaciones como elemento de garantía de seguridad, desarrollo y bienestar como sociedad.

La complejidad tecnológica y normativa que están alcanzando estas instalaciones es muy grande. Entran en juego múltiples valores directamente cuantificables, como la iluminancia y la eficiencia energética, y otros más complejos y subjetivos como el deslumbramiento o la contaminación lumínica.

\section{2.- Instalaciones de alumbrado público en España}

La iluminación constituye un porcentaje muy significativo del consumo energético de un país, según datos de la Agencia Internacional de la Energía (en adelante IEA), el 19\% de la generación eléctrica del planeta se dedica a la iluminación. Del mismo modo, datos del Ministerio de Industria, Turismo y Comercio Español nos colocan a la cola de la eficiencia energética en Europa al afirmar que [1]:

- El alumbrado público supone el 1,5\% del total de la energía consumida en España. En las ciudades, supone en la actualidad entre el $50 \mathrm{y}$ el $60 \%$ del su consumo energético total.

- $\quad$ El gasto en alumbrado público supone cerca de 450 millones de euros al año. Más de la mitad de la energía gastada se pierde emitiendo hacia el cielo, constituyendo una fuente de contaminación lumínica importantísima, o se desperdicia iluminando fachadas, vegetación o zonas 'muertas'.

- Este gasto crece en torno a un $4,7 \%$ anual y el consumo de energía un $2,6 \%$. 
Las lámparas de tecnología tradicional ofrecen sus datos característicos en lúmenes (Tabla 1), dado que emiten en casi la totalidad de direcciones del espacio y la luz que proyectan sobre un plano específico depende fuertemente del tipo de luminaria donde se instalan.

Los equipos de iluminación LED, por el propio modelo de emisor, emiten únicamente en un hemisferio y son mucho menos dependientes de difusores y ópticas para conducir la luz hacia el plano de trabajo. De este modo, con una emisión menor en lúmenes globales es capaz de conseguir valores en iluminancia más elevados.

Esta capacidad, junto con tiempos de vida muy elevados, alta robustez mecánica, capacidad de seleccionar la temperatura de color emitiendo luz blanca y de usar mecanismos de regulación y telegestión están fomentando que se proponga como la tecnología más adecuada para este tipo de instalaciones en la actualidad como se desprende de los datos del capítulo 4 de este trabajo.

\begin{tabular}{|c|c|c|}
\hline Sistema de alumbrado & Vida útil & Rendimiento \\
\hline \multicolumn{3}{|l|}{ INCANDESCENCIA } \\
\hline Convencional & $1.000 \mathrm{~h}$ & $12-18 \mathrm{~lm} / \mathrm{W}$ \\
\hline Halógena & $2.000 \mathrm{~h}$ & $18-22 \mathrm{~lm} / \mathrm{W}$ \\
\hline \multicolumn{3}{|l|}{ LÁMPARA DE DESCARGA } \\
\hline Vapor de mercurio baja presión & $5.000-15.000 h$ & $38-91 \mathrm{~lm} / \mathrm{W}$ \\
\hline Vapor de mercurio alta presión (VMAP) & $8.000 \mathrm{~h}$ & $40-60 \mathrm{~lm} / \mathrm{W}$ \\
\hline VMAP - luz de mezcla & $6.000 \mathrm{~h}$ & $20-60 \mathrm{~lm} / \mathrm{W}$ \\
\hline VMAP - halogenuros metálicos & $9.000 \mathrm{~h}$ & $60-95 \mathrm{Im} / \mathrm{W}$ \\
\hline Vapor de sodio de baja presión & $6.000-8.000 h$ & $160-180 \mathrm{~lm} / \mathrm{W}$ \\
\hline Vapor de sodio de alta presión & $8.000-12.000 h$ & $130 \mathrm{~lm} / \mathrm{W}$ \\
\hline Lámpara fluorescente compacta & $8.000 \mathrm{~h}$ & $60 \mathrm{~lm} / \mathrm{W}$ \\
\hline \multicolumn{3}{|l|}{ PLASMA } \\
\hline Plasma & $30.000 \mathrm{~h}$ & $85 \mathrm{~lm} / \mathrm{W}$ \\
\hline \multicolumn{3}{|l|}{ ILUMINACIÓN DE ESTADO SÓLIDO } \\
\hline LEDs & $2100.000 \mathrm{~h}$ & $z 160 \mathrm{~lm} / \mathrm{W}$ (y creciendo) \\
\hline
\end{tabular}

Tabla 1: Resumen de tiempos de vida y eficiencias promedias 
WPSReview International on Sustainable Housing and Urban Renewal

(RI-SHUR)

de las tecnologías de iluminación [1]

\section{1.- Legislación y normativa general}

Todos los productos incluidos en este ámbito están sometidos obligatoriamente al cumplimiento de la siguiente legislación, preferentemente vinculada a la seguridad eléctrica para las personas y la compatibilidad electromagnética con otros equipos:

- $\quad$ Directiva de Baja Tensión- 2006/95/CEE. Relativa a la aproximación de las Legislaciones de los Estados Miembros sobre el material eléctrico destinado a utilizarse con determinados límites de tensión.

- Directiva de Compatibilidad Electromagnética- 2004/108/CEE. Relativa a la aproximación de las Legislaciones de los estados miembros en materia de compatibilidad electromagnética y por la que se deroga la Directiva 89/336/CE.

- Directiva ROHS 2011/65/UE. Relativa a las restricciones a la utilización de determinadas sustancias peligrosas en aparatos eléctricos y electrónicos.

- Real Decreto 154/1995, sobre exigencias de seguridad del material eléctrico destinado a ser utilizado en determinados límites de tensión y su Guía de Interpretación.

- Real Decreto 842/2002 por el que se aprueba el Reglamento Electrotécnico de Baja Tensión y sus instrucciones Técnicas Complementarias ITC-BT-01 a ITC-BT-51.

- Reglamento 245/2009, de la Comisión de 18 de marzo por el que se aplica la Directiva 2005/32/CE del Parlamento Europeo relativo a los requisitos de diseño ecológico, para lámparas, balastos y luminarias.

- Directiva de Ecodiseño 2009/125/CE. Por la que se instaura un marco para el establecimiento de requisitos de diseño ecológico aplicables a los productos relacionados con la energía.

- Reglamento No 1194/2012 de la por el que se aplica la Directiva de Ecodiseño- 2009/125/ CE a las lámparas direccionales, lámparas LED y sus equipos. 
WPSReview International on Sustainable Housing and Urban Renewal

(RI-SHUR)

- Reglamento 874/2012 de 12 de julio de 2012 por el que se complementa la Directiva 2010/30/UE del Parlamento Europeo y del Consejo en lo relativo al etiquetado energético de las lámparas eléctricas y las luminarias.

Junto a esto necesitan cumplir con los requisitos para obtener el marcado CE, que indica que todo elemento o componente que exhibe dicho marcado debe cumplir con las normas armonizadas indicadas en la tabla 2.

\begin{tabular}{|c|c|c|}
\hline Familia de Producto & $\begin{array}{l}\text { Seguridad } \\
\text { Eléctrica }\end{array}$ & $\begin{array}{l}\text { Compatibilidad } \\
\text { Electromagnética }\end{array}$ \\
\hline $\begin{array}{l}\text { Lámpara LED con fuente } \\
\text { de alimentación incluida }\end{array}$ & $\begin{array}{l}\text { UNE-EN 60.598-1 } \\
\text { UNE-EN 60.598-2-3 } \\
\text { UNE-EN } 62.031\end{array}$ & $\begin{array}{c}\text { UNE-EN 55.015 } \\
\text { UNE-EN 61.000-3-2 } \\
\text { UNE-EN 61.000-3-3 } \\
\text { UNE-EN } 61.547\end{array}$ \\
\hline
\end{tabular}

Tabla 2: Normas Armonizadas de obligado cumplimiento requeridas para el marcado $\mathrm{CE}$ [2]

Junto con los elementos de este listado, existen un número mucho más extenso de normas vinculadas a este tipo de productos no de obligado cumplimiento. Para servir de referencia a fabricantes y técnicos municipales, el IDAE junto con el CEI [3] han generado un listado de esta normativa. Este documento se está usando en muchos concursos como requisitos mínimos de participación en un intento de aumentar, mucho, la calidad de las instalaciones de iluminación.

\section{2.- Protección de la Calidad del Cielo Nocturno}

Además del conjunto de normas del apartado anterior, tiene una importancia específica alta la regulación de la protección del cielo nocturno. Las emisiones 
directas hacia el cielo, así como la radiación reflejada en las superficies iluminadas, al difundirse y reflejarse en los gases y partículas en suspensión de la atmósfera, originan un resplandor luminoso nocturno, dando lugar a que se incremente el brillo del fondo natural del cielo, dificultando las observaciones astronómicas de los objetos celestes y alterando la vida del ecosistema natural, constituyendo la denominada "contaminación lumínica" [4]. Este control es una competencia transferida a las comunidades autónomas y está siendo desarrollada normativamente de manera independiente por cada gobierno [5] (ver tabla 3):

- Limitando la emisión hacia el hemisferio superior de la luminaria

- $\quad$ Eligiendo las de mayor rendimiento

- Implantando el menor número de puntos de luz que permita alcanzar los niveles requeridos

- Apagado, a determinadas horas de la noche, de iluminaciones ornamentals

- Disminución de los niveles luminosos, sin distorsionar la uniformidad, en las horas en que la intensidad del tráfico desciende substancialmente, siempre y cuando quede garantizada la seguridad de los usuarios. 


\section{WPSReview International on Sustainable Housing and Urban Renewal}

\section{(RI-SHUR)}

\begin{tabular}{|c|c|c|}
\hline & LEY & DECRETO \\
\hline Cataluña & $\begin{array}{l}\text { Ley } 6 / 2001 \text {, de } 31 \text { de } \\
\text { mayo, de ordenación } \\
\text { ambiental del } \\
\text { alumbrado para la } \\
\text { protección del medio } \\
\text { nocturno }\end{array}$ & $\begin{array}{l}\text { Decreto } 82 / 2005 \text {, de } 3 \text { de mayo, } \\
\text { por el que se aprueba el } \\
\text { Reglamento de desarrollo de la } \\
\text { Ley } 6 / 2001 \text {, de } 31 \text { de mayo, de } \\
\text { ordenación ambiental del } \\
\text { alumbrado para la protección } \\
\text { del medio nocturno }\end{array}$ \\
\hline Islas Baleares & $\begin{array}{l}\text { Ley } 3 / 2005 \text {, de } 20 \text { de } \\
\text { abril, de protección } \\
\text { del medio nocturno de } \\
\text { las Illes Balears }{ }^{(1)}\end{array}$ & - \\
\hline Navarra & $\begin{array}{l}\text { Ley Foral } 10 / 2005 \text {, de } \\
9 \text { de noviembre, de } \\
\text { ordenación del } \\
\text { alumbrado para la } \\
\text { protección del medio } \\
\text { nocturno }\end{array}$ & $\begin{array}{l}\text { Decreto Foral 199/2007, de } 17 \\
\text { de septiembre, por el que se } \\
\text { aprueba el Reglamento de } \\
\text { desarrollo de la Ley Foral } \\
10 / 2005 \text {, de } 9 \text { de noviembre, de } \\
\text { ordenación del alumbrado para } \\
\text { la protección del medio } \\
\text { nocturno }\end{array}$ \\
\hline Cantabria & $\begin{array}{l}\text { Ley de Cantabria } \\
6 / 2006 \text {, de } 9 \text { de junio, } \\
\text { de prevención de la } \\
\text { contaminación } \\
\text { luminica }\end{array}$ & - \\
\hline Andalucia & $\begin{array}{l}\text { Ley } 7 / 2007 \text {, de } 9 \text { de } \\
\text { julio, de gestión } \\
\text { integrada de la } \\
\text { calidad ambiental } \\
\text { (Sección } 3^{\mathrm{a}} \text {, Capitulo } \\
\text { II, Titulo IV) }\end{array}$ & $\begin{array}{l}\text { Borrador en } \\
\text { administrativo }\end{array}$ \\
\hline
\end{tabular}

Tabla 3: Normas de rango autonómico. Legislación desarrollada por gobiernos regionales de España para la protección del medio nocturno.

\section{3.- Metodología}

Para intentar formalizar un análisis adecuado se ha recopilado la información de concursos repartidos por toda la península. Se ha utilizado la información de 53 concursos (ver figura 1) aunque es importante destacar que no todos se publican organizados de la misma manera ni tienen el mismo contenido. Algunos de ellos simplemente te indican las cláusulas que debe tener el contrato junto al presupuesto de licitación y los criterios de adjudicación en el 
WPSReview International on Sustainable Housing and Urban Renewal

(RI-SHUR)

"Pliego de Cláusulas Administrativas"; y las condiciones que deben tener las lámparas y luminarias propuestas y algunos de sus criterios técnicos en el "Pliego de Condiciones Técnicas". Sin embargo, una de las opciones por la que los ayuntamientos suelen optar es la realización de una auditoría energética o proyecto de eficiencia energética. Este tipo de proyectos suelen incluir información sobre: descripción de las instalaciones; estudios lumínicos; cálculos energéticos; desglose de presupuestos; etc.

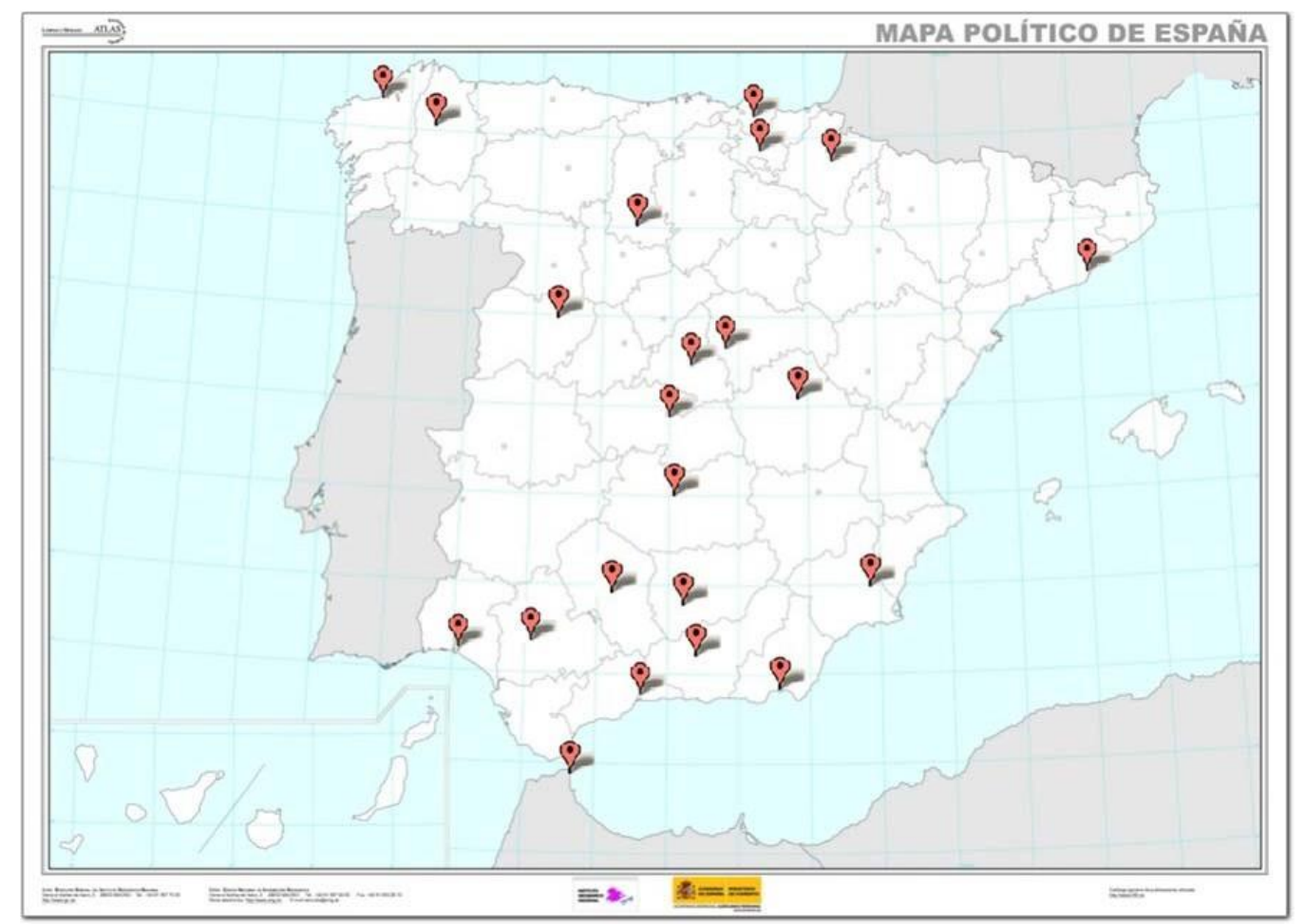

Figura 1: Localización de los concursos públicos analizados

22 de los 53 concursos adjuntan una auditoría o proyecto energético, facilitando en la mayoría de los casos una mayor cantidad de información de las instalaciones actuales que el resto. 
De los 53 concursos analizados solo 7 de ellos buscaban contratar a una Empresas de Servicios Energéticos (ESEs) para realizar todo lo relacionado con ahorro energético del municipio durante los años que se le contraten. El resto eran de suministro de equipos o suministro e instalación.

\section{4.- Análisis de datos}

En los concursos públicos de esta naturaleza debería ser imprescindible realizar un estudio detallado de la situación previa de las instalaciones de alumbrado público. Conocer el tipo de tecnología a sustituir, el estado de las instalaciones, la potencia instalada y contratada, el número de horas de uso anual y el respectivo consumo energético, el factor de potencia de toda la instalación, etc., es importante para poder valorar la mejora en eficiencia energética que se está planificando, optimizar los contratos de electricidad y verificar lo ahorros económicos.

En este apartado se realizarán una serie de análisis de los parámetros más destacables en las instalaciones actuales de los municipios que salen a concurso.

\section{1.- Número de puntos de luz por concurso}

Para estimar la amplitud de los concursos se han agrupado por cantidad de puntos de luz a sustituir en la localidad como muestra la figura 2. Prácticamente el $57 \%$ de los concursos estudiados son de municipios muy pequeños que renuevan menos de 500 puntos de luz. 


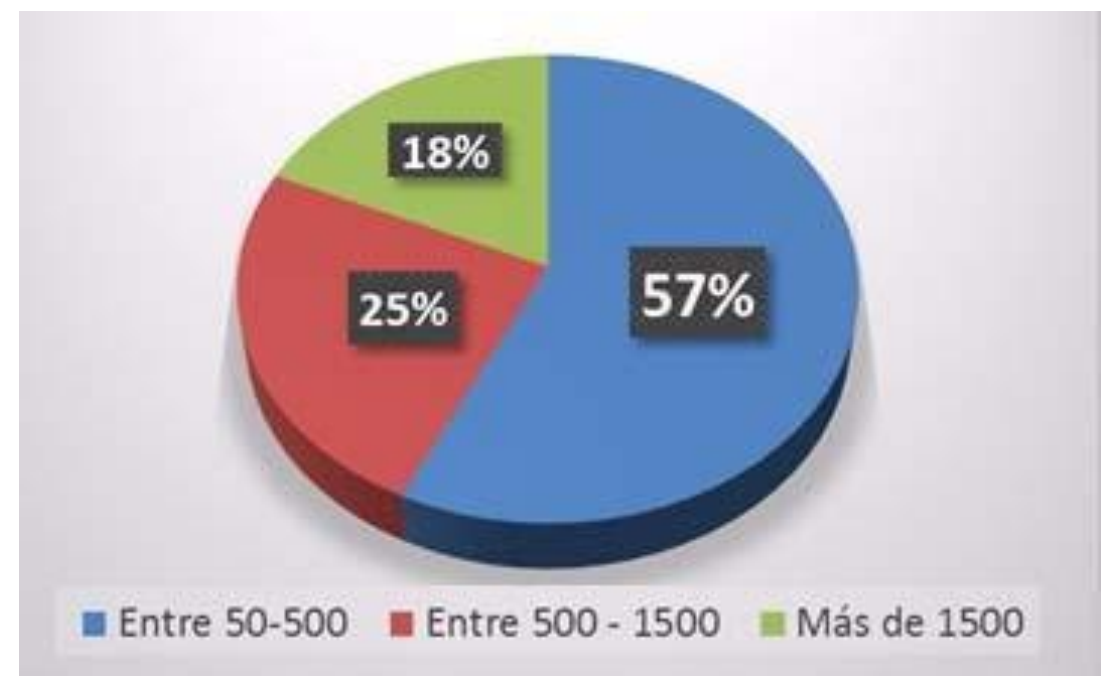

Figura 2: Número de puntos de luz tratados por concurso.

\section{2.- Tecnología de iluminación a sustituir}

Actualmente, existen todo tipo de instalaciones. Los concursos priman sustituir las menos eficientes por el auge de otras tecnologías de mayor rendimiento. La figura 3 muestra las tecnologías predominantes en las instalaciones actuales. Las más utilizadas son las lámparas VSAP, los halogenuros metálicos (en adelante HM) y el vapor de mercurio (en adelante VM). Se observa que prácticamente quedan muy pocas lámparas incandescentes y que están tendiendo a desaparecer. Ya existen luminarias LED usadas en pruebas pilotos o proyectos preliminares para verificar la tecnología. 


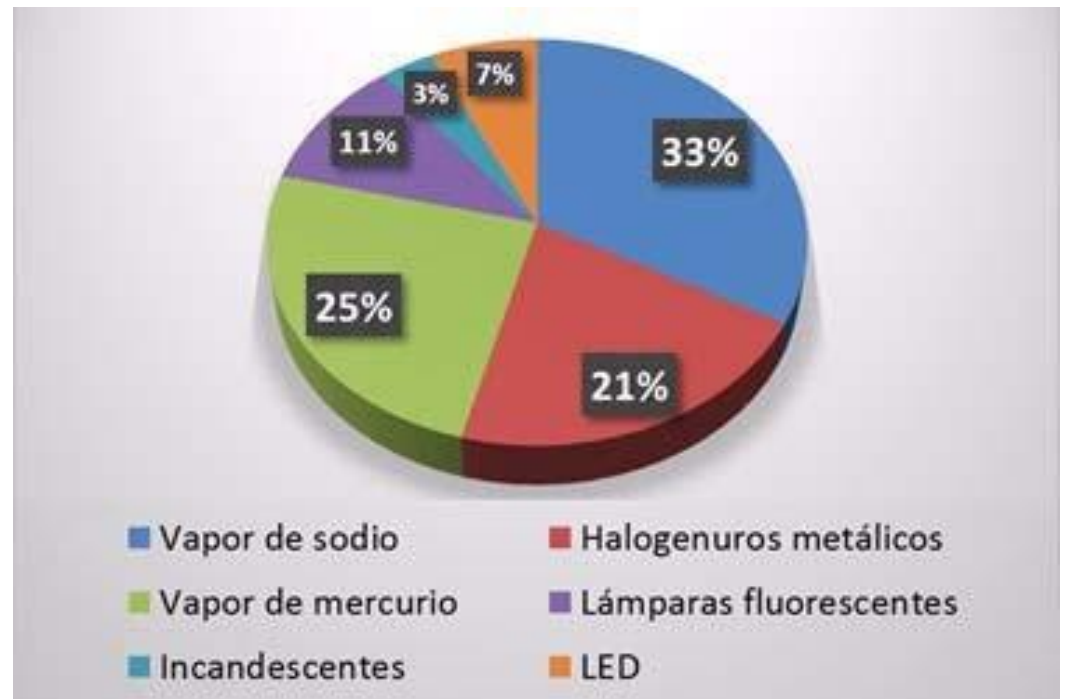

Figura 3: Tecnologías de iluminación que predominan actualmente

\section{3.- Potencia instalada y consumo energético}

La mitad de los concursos no aportan información alguna sobre el consumo previo de su instalación en la actualidad. Los datos de los concursos que si lo detallan se muestran en la figura 4.

En la mayoría de los casos no existe ningún tipo de regulación de flujo lumínico o de control, lo que conlleva a un exceso de luz durante la noche hasta la hora de apagado. 


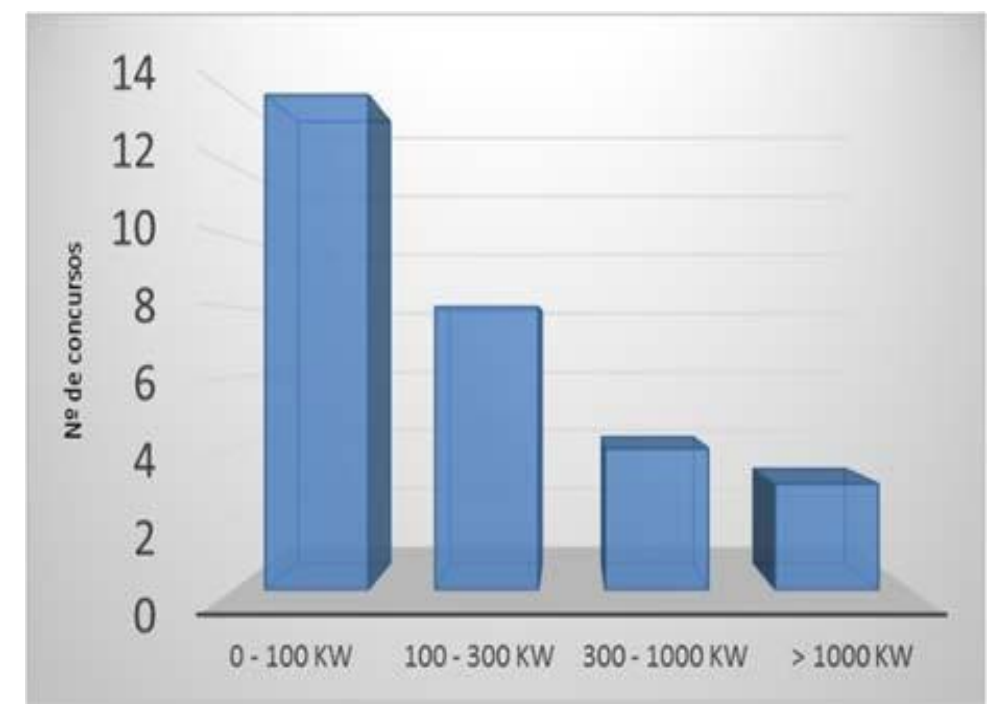

Figura 4: Potencia instalada $(\mathrm{kW})$ previa de los concursos analizados

\section{5.- Análisis de las propuestas de mejora}

En este apartado se analizan los parámetros fundamentales de las propuestas de cambio que se realizan en estos concursos.

\section{1.- Tecnología de iluminación}

La figura 5 muestra como la tecnología LED es la preferencia fundamental de actualización. Los motivos que se expresan son, fundamentalmente, las expectativas de ahorros energéticos muy elevados y minimizar el flujo hemisférico superior instalado ( $F H S i n s t)$, que no puede ser superior a ciertos valores (dependiente de la clasificación de sensibilidad ambiental de la zona declarada por la comunidad autónoma o el ayuntamiento).

Las tecnologías de vapor de sodio y halogenuros metálicos se mantienen principalmente en ampliaciones de instalaciones donde se quiere mantener una misma estética y funcionalidad. 


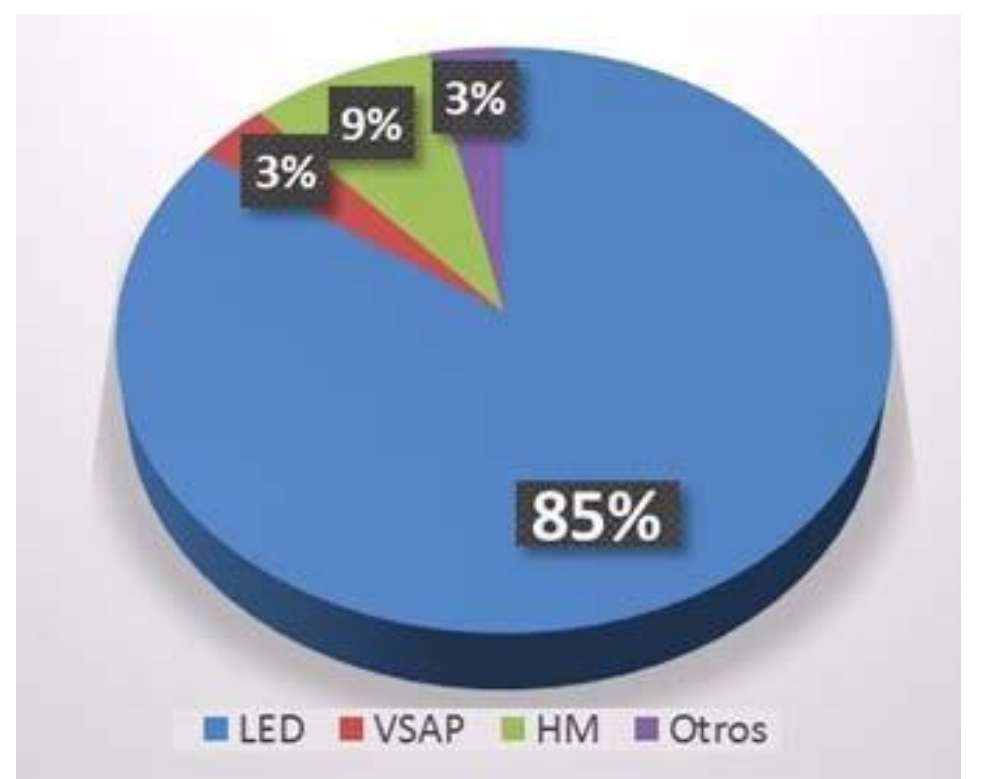

Figura 5: Tecnología de iluminación con mayor demanda

\section{2.- Telegestión y control}

Todas las propuestas analizadas exigen, al menos, un sistema de regulación lumínica básico y, en ocasiones prescriben instalar sistema de "Telegestión", que permiten realizar funciones de supervisión y gestión sobre una instalación consumidora de energía eléctrica (los centros de mando) y, en los casos más complejos, controlar las luminarias de manera independiente. La mayor inversión en la adquisición de estos sistemas es justifica en base a mayores ahorros eléctricos, minimización del mantenimiento y mayor control sobre la instalación para atender a eventos especiales (fiestas, obras,...). 


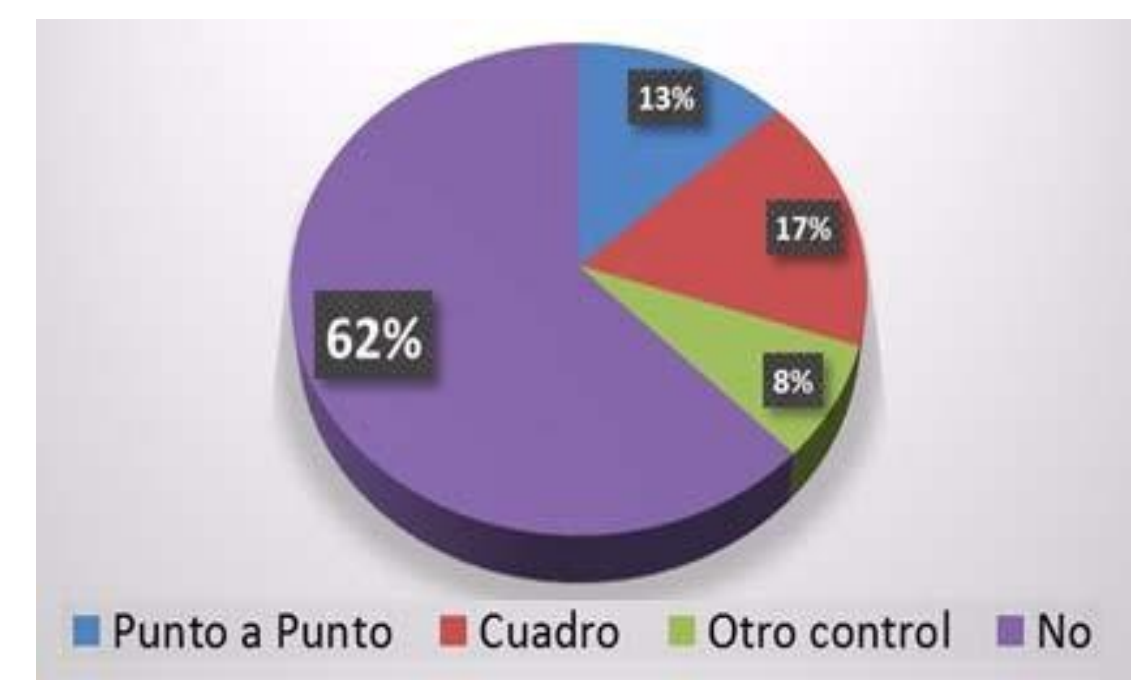

Figura 6: Sistemas de telegestión en las propuestas de mejora

La figura 6 muestra cómo en la mayoría de los concursos no se plantea la instalación de un sistema de Telegestión, debido al coste que esto supone y a los mayores tiempos de amortización que necesitan. Sin embargo, en las capitales de comunidades autónomas y grandes ciudades cada vez se extienden más.

\section{3.- Clasificación de vías públicas}

\subsection{1.- Clasificación del alumbrado}

La figura 7 muestra las clasificaciones de los viales que se plantean renovar en estos procesos. Las clases más comunes son la ME4b, vías principales y circunvalaciones en la que se exigen unos criterios de iluminación mayor y, la clase S2 que se suele utilizar para calles residenciales. 


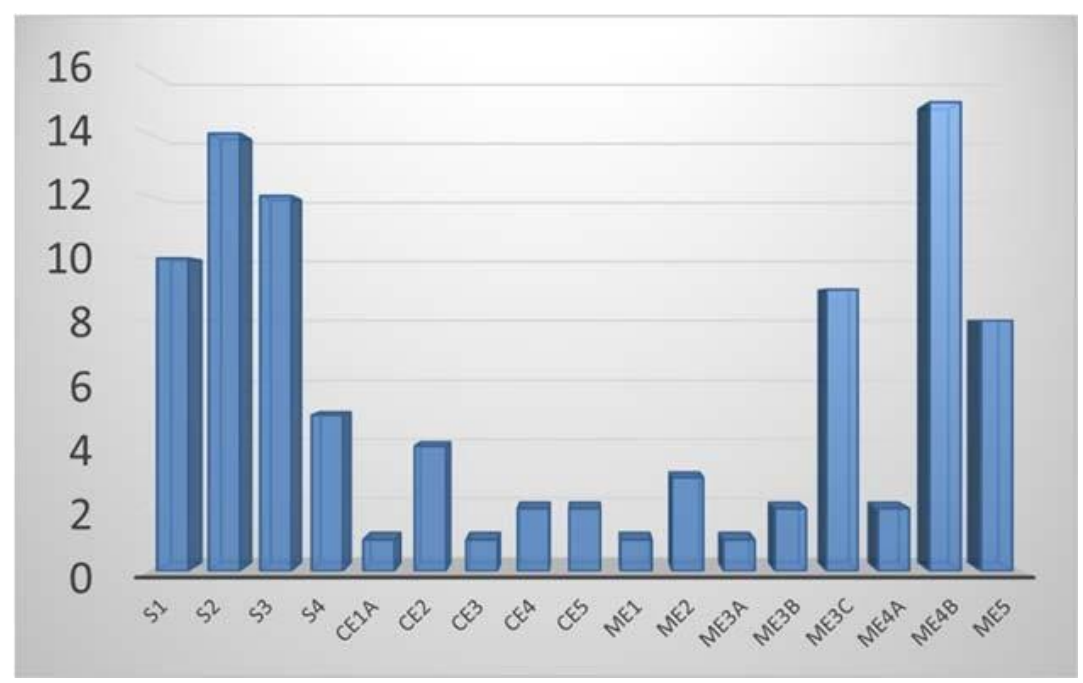

Figura 7: Clase de alumbrado más común en concursos

\subsection{2.- Clasificación energética}

La clasificación energética mide la eficiencia energética de una instalación de alumbrado

exterior. El tipo A, el nivel más alto, es lo que se exige en todos los concursos.

\section{4.- Parámetros de la instalación}

\subsection{1.- Estimación de la nueva potencia instalada}

A fin de realizar un estudio de la viabilidad económica y el ahorro de consumo energético, se suele realizar una estimación de la potencia que deben de tener las nuevas luminarias que se van a instalar. La tabla 4 detalla una comparativa de las potencias instaladas actuales, las propuestas de mejora y la reducción estimada de potencia sustituyendo tecnologías convencionales por sistemas LED. 
(RI-SHUR)

\begin{tabular}{|c|c|c|c|}
\hline \multicolumn{4}{|c|}{ Potencia instalada (kW) } \\
\hline Concurso & Actual & $\begin{array}{l}\text { Propuesta de } \\
\text { mejora }\end{array}$ & $\begin{array}{l}\text { Porcentaje } \\
\text { reducido }\end{array}$ \\
\hline $\begin{array}{l}\text { Alameda de la Sagra } \\
\text { (Toledo) }\end{array}$ & 111,39 & 31,72 & $71,5 \%$ \\
\hline $\begin{array}{l}\text { Alegría-Dulantzi y Egileta } \\
\text { (Álava) }\end{array}$ & 87,27 & 28,13 & $67,8 \%$ \\
\hline Berga (Barcelona) & 480,79 & 155,28 & $67,7 \%$ \\
\hline Bujalaro (Guadalajara) & 11,00 & 6,70 & $39,1 \%$ \\
\hline Camas (Sevilla) & 15,50 & 10,53 & $32,0 \%$ \\
\hline $\begin{array}{l}\text { Carrión de los condes } \\
\text { (Palencia) }\end{array}$ & 189,85 & 68,87 & $63,7 \%$ \\
\hline Casarabonela (Málaga) & 51,80 & 25,51 & $50,8 \%$ \\
\hline Casariche (Sevilla) & 76,92 & 19,82 & $74,2 \%$ \\
\hline Cedeira (A Coruña) & 9,00 & 4,20 & $53,3 \%$ \\
\hline Ceuta & 32,51 & 17,23 & $47,0 \%$ \\
\hline Coín (Málaga) & 312,12 & 83,78 & $73,2 \%$ \\
\hline Ginés (Sevilla) & 357,07 & 136,91 & $61,7 \%$ \\
\hline Macael (Almería) & 166,35 & 62,08 & $62,7 \%$ \\
\hline Orduña (Vizcaya) & 165,80 & 63,76 & $61,5 \%$ \\
\hline $\begin{array}{l}\text { Parque fluvial de } \\
\text { Fuengirola }\end{array}$ & 45,87 & 16,52 & $64,0 \%$ \\
\hline Pinto (Madrid) & 184,35 & 37,26 & $79,8 \%$ \\
\hline Rielves (Toledo) & 24,64 & 9,58 & $61,1 \%$ \\
\hline $\begin{array}{l}\text { San Martín de la Vega } \\
\text { (Madrid) }\end{array}$ & 147,48 & 36,55 & $75,2 \%$ \\
\hline Santander & 10.378 & 2075,60 & $80,0 \%$ \\
\hline Yecla (Murcia) & 161,36 & 59,72 & $63,0 \%$ \\
\hline
\end{tabular}

Tabla 4: Estimación del ahorro en potencia instalada (kW) 


\subsection{2.- Factor de potencia}

La normativa actual y las compañías eléctricas prestan especial atención al factor de potencia de las instalaciones, un valor elevado hace que la potencia aparente de la misma descienda y, por consiguiente, los requisitos de sección de cables se reducen.

La documentación que se aporta en los concursos públicos no indica en ninguno de los casos el factor de potencia que tienen las instalaciones actuales, pero en la mayoría de los casos sí que se incluye el de la propuesta de mejora. Este valor suele ser 0,9 y los casos más exigentes 0,95.

\section{5.-Presupuesto de licitación}

Existen todo tipo de concursos, desde la sustitución de unas pocas luminarias de ciertas calles de un pueblo, hasta la renovación total de las instalaciones del municipio. Sin embargo, hay una cierta tendencia que indica que la mayor parte de los concursos se realizan para pequeños municipios de manera independiente y sin agruparse y, por tanto, las inversiones económicas no son especialmente elevadas.

La figura 8 muestra el presupuesto invertido en los concursos estudiados. La gran mayoría están en torno a los $500.000 €$, pero varios concursos pertenecientes a municipios más grandes, hacen subir la media hasta un valor de 1.400.000€. 


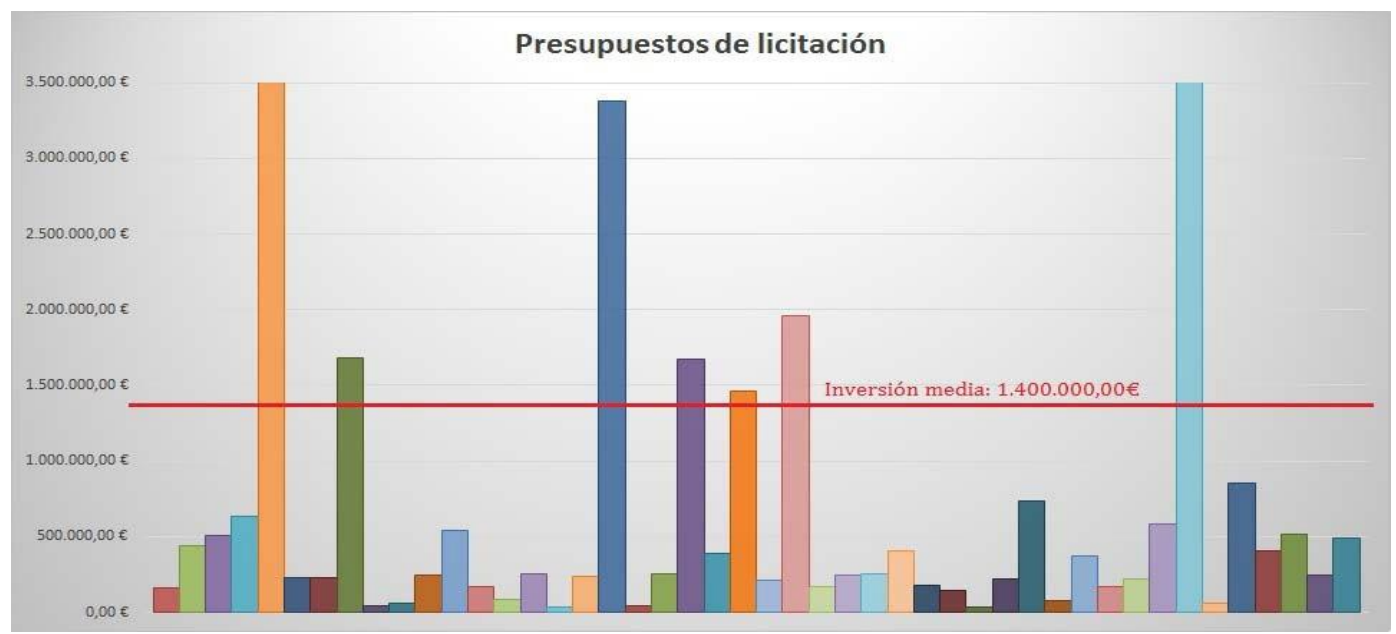

Figura 8: Presupuestos de licitación e inversión media

5.6.- Consumo energético y ahorro annual

El consumo energético junto con la potencia eléctrica contratada son los dos elementos con más peso en los costes de explotación de estas instalaciones. Una de las ventajas que proporcionan los sistemas LED es que necesitas instalar mucha menos potencia para obtener unos resultados similares 0 mejores por lo que se puede ahorrar en estos dos elementos de la factura eléctrica.

Muchos de estos concursos exigen instalar una potencia específica de luminarias LED, sin permitir que los licitadores propongan alcanzar un ahorro mayor usando potencias menores a las exigidas con unos cálculos y simulaciones justificativas asociadas. Estas condiciones parecen muy contradictorias si el objetivo final deseado es el ahorro de energía cumpliendo con los criterios de iluminación exigidos.

\section{6.-Simulaciones lumínicas}

La realización de cálculos lumínicos a través de softwares de simulación informática que permitan obtener resultados y de esta manera comprobar que se cumple con la normativa es uno de los requerimientos que recomienda exigir el IDAE en la planificación de instalaciones. 


\section{WPSReview International on Sustainable Housing and Urban Renewal}

(RI-SHUR)

En la mayoría de concursos que tienen asociados proyectos de eficiencia energética o anexos de cálculos lumínicos, se destina un apartado para estos cálculos. Sin embargo, la realidad que hemos encontrado es que hay un número considerable de concursos que no aportan dichos cálculos y muchos de ellos son de fiabilidad cuestionable (parámetros de luminarias fuera de los rangos tecnológicos actuales, factores de mantenimiento mal calculados,)

Estos programas generan documentos en los que se muestran toda la información de las simulaciones, como muestra la figura 9.

Lista del recuadro de evaluación

2 Acera 2

Longitud: $25.000 \mathrm{~m}$, Anchura: $2.000 \mathrm{~m}$

Trama: $10 \times 3$ Puntos

Elemento de la vía pública respectivo: Acera 2.

Clase de iluminación seleccionada: CE5

(No se cumplen todos los requerimientos fotométricos.)

Valores reales según cálculo:

Valores de consigna según clase:

Cumplido/No cumplido:

3 Recuadro de evaluación Calzada 1

Longitud: $25.000 \mathrm{~m}$, Anchura: $10.000 \mathrm{~m}$

Trama: $10 \times 6$ Puntos

Elemento de la vía pública respectivo: Calzada 1.

Revestimiento de la calzada: R3, q0: 0.070

Clase de iluminación seleccionada: ME5

(Se cumplen todos los requerimientos fotométricos.)

Valores reales según cálculo:

Valores de consigna según clase:

$\mathrm{L}_{\mathrm{m}}\left[\mathrm{cd} / \mathrm{m}^{2}\right]$
0.61
$\geq 0.50$

U0
0.44
$\geq 0.35$

UI

TI [\%]

20.35

20.40

Cumplido/No cumplido:

4 Recuadro de evaluación Carril de estacionamiento 2

Longitud: $25.000 \mathrm{~m}$, Anchura: $2.500 \mathrm{~m}$

Trama: $10 \times 3$ Puntos

Elemento de la vía pública respectivo: Carril de estacionamiento 2 .

Clase de iluminación seleccionada: CE5

(Se cumplen todos los requerimientos fotométricos.)

Valores reales según cálculo:

Valores de consigna según clase:

$E_{m}[\mathrm{~b}]$

10.38

27.50

Cumplido/No cumplido: 
WPSReview International on Sustainable Housing and Urban Renewal

(RI-SHUR)

Figura 9: Ejemplo de resultados de simulación en DIALux

Estos programas son sistemas internacionales que se configuran en base a normas UNE $i$ ?, pero que no recogen las normativas nacionales propias. El RD1980/2008 vigente en España prohíbe que una instalación sobrepase el $120 \%$ del máximo establecido por la normativa de una vía. Así el caso de la figura 9 se indica como correcto a pesar de sobrepasar este factor de ponderación, por lo que se estaría incumpliendo este Real Decreto.

Un aumento en las exigencias de estandarización de este tipo de documentos evitaría el incumplimiento de la normativa y facilitaría la comparativa de diferentes propuestas de instalaciones.

\section{7.- Análisis de desarrollos específicos de concursos públicos}

Un porcentaje alto de los concursos que se sacan a licitación pública en España son impugnados. Las alegaciones que se presentan son de muchos tipos: por defectos de forma, por fallos en los datos de base o por discrepancia con la adecuación de algunos criterios selectivos determinados para el mismo que hacen que no cumplan con todos los requisitos de la legislación vigente, tal y como corroboran las decisiones de los tribunales de arbitraje, fallando en garantizar la igualdad de oportunidades de optar a estos contratos públicos.

En este apartado desarrollamos una breve crítica propia de la adecuación legal de algunos de los concursos analizados.

\subsection{Concurso público de Rielves}

El apartado "5.3. Valoración Económica de las mejoras propuestas" del pliego de cláusulas administrativas de este concurso detalla un resumen de los presupuestos para las medidas correctoras aplicables que incluye la suma de los importes y se le aplica un porcentaje de impuestos de valor añadido erróneo. Utilizar un IVA del $18 \%$ en lugar del $21 \%$ es un fallo grave en estos

WPS RI-SHUR, n5, 2017, vol.1, ISSN: 2387-1768 
WPSReview International on Sustainable Housing and Urban Renewal

(RI-SHUR)

procesos puesto que todo el estudio económico está basado en estos presupuestos. Este error se repite en todos los documentos del concurso que hacen referencias a parámetros económicos (ver figura 10)

\section{RESUMEN}

Medidas para el cumplimiento del REBT.

Figura 10: Porcentaje de IVA aplicado erróneo (18\%)

\section{2.- Concurso público de Orduña}

Este pliego de cláusulas administrativas muestra unos criterios de adjudicación basados en una serie de ensayos propios por parte del ayuntamiento para medir los parámetros de muestras de las luminarias presentadas al concurso (Ver figura 11). Sin embargo, realizar esos ensayos en un principio de igualdad y en base a los criterios normativos adecuados requiere de un laboratorio especializado con herramientas de medición precisas (p.e. para poder obtener un resultado entre 0,99 y 1 de factor de potencia). Además, hay que tener en cuenta que un valor de factor de potencia entre 0,99 y 0,95 solo se premia con 2 puntos, a diferencia de los 10 puntos que sería la valoración máxima. Por otro 
lado, la normativa exige realizar las medidas en los equipos tras alcanzar valores estables de funcionamiento ya que este sería el valor real en la instalación a la hora de su uso y no justo tras su encendido. Medir la potencia en el instante de encendido no aporta ninguna información significativa, ya que este es un pequeño transitorio que no será válido pasado poco tiempo.

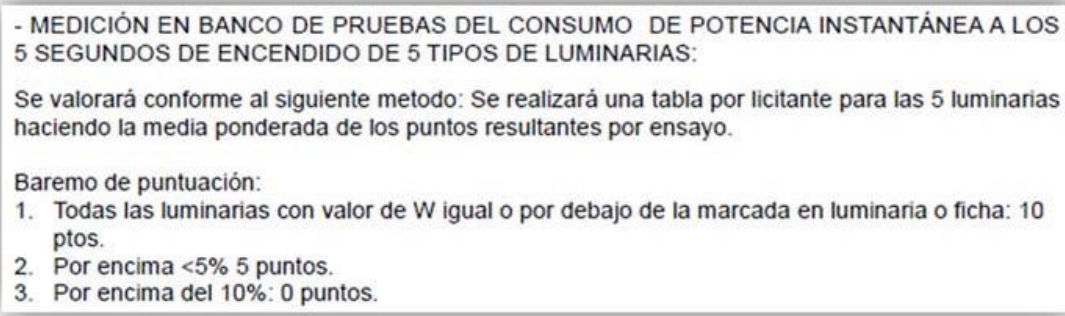

Figura 11: Criterio de baremación en el concurso de Orduña [6]

\section{3.- Concurso Público de Dos Hermanas}

A este concurso se le realizó un recurso especial en materia de contratación contra el "Pliego de Cláusulas Administrativas" particulares (en adelante PCA) y el de "Prescripciones Técnicas" (en adelante PPT) que rigen el contrato de este concurso.

Se esgrimía que dichos pliegos restringían las posibilidades de la empresa de acceder a licitación y dificultaba la libre competencia vulnerando el principio establecido en el apartado 2 del artículo 117 del Texto Refundido para las 
WPSReview International on Sustainable Housing and Urban Renewal

(RI-SHUR)

Leyes de Contratación del Sector Público (en adelante TRLCSP), conforme al cual las prescripciones técnicas deberían permitir la igualdad entre licitadores.

Se argumentaba que el PPT no realizaba la definición del objeto del contrato mediante especificaciones técnicas, sino mediante la referencia a una marca de fabricante concreto (ver figura 12). Esta posibilidad solo es admisible cuando se apliquen sistemas de referencia técnicas elaboradas por organismos de homologación y no incluía la expresión "o equivalente", con el objeto de permitir presentar ofertas con otro tipo de productos que pudiesen cumplir las condiciones técnicas expuestas.

"4.3 Características de los grupos ópticos para luminarias IQV.

Grupo óptico ERP775 ECO/8350/NW PSDD OFR6 DD27 temperatura de color $4.000 \mathrm{~K}$ con programación de regulación de flujo".

Figura 12: Clausula objeto de impugnación del concurso de Dos Hermanas [7]

Para que un contrato pueda referenciar a una marca se deben cumplir las siguientes tres condiciones simultáneamente:

a) Que la referencia a una marca, patente, tipo o producción determinados esté justificada

por el objeto del contrato.

b) Que la entidad adjudicadora no tenga la posibilidad de dar otra descripción del objeto del contrato a través de especificaciones suficientemente precisas para todos los potenciales licitadores.

c) Que la indicación de la marca esté acompañada de la mención «0 equivalente», 
WPSReview International on Sustainable Housing and Urban Renewal

(RI-SHUR)

Estas condiciones que son acumulativas y que deberá demostrarse que se cumplen las tres. Lo que no ocurre en este caso quebrando los principios de igualdad contenidos en los artículos 1 y 139 del TRLCSP.

\section{4.- Otros casos}

A modo de resumen, se ha elaborado la tabla 5 que muestra incidencias en algunos de los diferentes concursos estudiados en este trabajo.

\begin{tabular}{|c|c|}
\hline Concurso público & Elementos problemáticos \\
\hline Abanto y Ciérvana (Vizcaya) & Falta de información y/o anexos \\
\hline Arteixo (A Coruña) & Aceras con distinta clasificación \\
\hline Cabanillas (Navarra) & $\begin{array}{l}\text { Movilización a la localidad para } \\
\text { poder realizar oferta }\end{array}$ \\
\hline Casasimarro (Cuenca) & Falta de información y/o anexos \\
\hline Ceuta & $\begin{array}{l}\text { Incluir y exigir marcas concretas de } \\
\text { luminarias en los pliegos }\end{array}$ \\
\hline Dos hermanas (Sevilla) & $\begin{array}{l}\text { Incluir y exigir marcas concretas de } \\
\text { luminarias en los pliegos }\end{array}$ \\
\hline Guijuelo (Salamanca) & Falta de información y/o anexos \\
\hline Macael (Almería) & $\begin{array}{l}\text { Incluir y exigir marcas concretas de } \\
\text { luminarias en los pliegos }\end{array}$ \\
\hline Mijas (Málaga) & Falta de información y/o anexos \\
\hline Orduña (Vizcaya) & $\begin{array}{l}\text { Criterios de adjudicación } \\
\text { cuestionables }\end{array}$ \\
\hline Parque fluvial de Fuengirola & $\begin{array}{l}\text { Incluir y exigir marcas concretas de } \\
\text { luminarias en los pliegos }\end{array}$ \\
\hline Peligros (Granada) & $\begin{array}{l}\text { Movilización a la localidad para } \\
\text { poder realizar oferta }\end{array}$ \\
\hline Pinto (Madrid) & $\begin{array}{l}\text { Incluir y exigir marcas concretas de } \\
\text { luminarias en los pliegos }\end{array}$ \\
\hline Rielves (Toledo) & $\begin{array}{l}\text { Uso erróneo de } 18 \% \text { de IVA en } \\
\text { cálculos económicos }\end{array}$ \\
\hline Roquetas de mar (Almería) & $\begin{array}{l}\text { Incluir y exigir marcas concretas de } \\
\text { luminarias en los pliegos }\end{array}$ \\
\hline Solana (Ciudad Real) & $\begin{array}{l}\text { Certificados no oficiales de } \\
\text { empresas privadas (como ENEC) }\end{array}$ \\
\hline Talavera de la reina (Toledo) & $\begin{array}{l}\text { Certificados no oficiales de } \\
\text { empresas privadas (como ENEC) }\end{array}$ \\
\hline
\end{tabular}

Tabla 5: Elementos problemáticos encontrados en algunos concursos públicos analizados 


\section{8.- Conclusiones}

En este trabajo se pretende mostrar, con cierta generalidad, las condiciones actuales de las instalaciones de alumbrado público de nuestra geografía y cómo se está desarrollando la tendencia mundial de renovación y mejora de estas instalaciones a través de los concursos públicos en base a la irrupción de la tecnología LED.

Un alto número de instalaciones de alumbrado público exterior actualmente se encuentran en un estado significativamente deficiente con respecto a los últimos desarrollos de normativas (por ejemplo, la de protección de cielo nocturno) y, especialmente, por criterios de ahorro económicos. De este modo, se ha iniciado un proceso generalizado de renovación.

La mayor parte de los proyectos que salen a concurso pertenecen a municipios pequeños que requieren inversiones reducidas para renovar sus instalaciones. Este tipo de instalación necesitan, normalmente, requerimientos lumínicos bajos, por ello, la clase de alumbrado "S2" es una de las más pedidas en los pliegos.

Para que la información aportada por un concurso sea completa y permita renovaciones más eficientes y estimaciones de ahorros y periodos de amortización más realistas es importante que consten de una auditoría energética referida a la situación actual de las instalaciones, para que los regidores, los técnicos municipales y los licitadores tengan disponible esa información a la hora de realizar cálculos, estimaciones, presupuestos y ofertas.

La tecnología que más predominan es el vapor de sodio, sin embargo, en más del $85 \%$ de loscasos de propuestas de mejora analizados se pretende instalar tecnología LED.

Muy pocas instalación que pretende renovarse consta de algún tipo de regulación. En las propuestas de mejora el 30\% de los casos opta por añadir sistemas de control y regulación remota, el resto instalará sistemas más básicos de regulación, sobre todo para las horas nocturnas. 
WPSReview International on Sustainable Housing and Urban Renewal

(RI-SHUR)

Con el fin de reducir al mínimo las pérdidas y optimizar las facturas eléctricas, no solo en el consumo o la potencia contratada sino también en las corrientes reactivas, todos los concursos exigen que el sistema completo tenga un factor de potencia mayor de 0,9.

En cuanto a la elaboración de los concursos en sí, se detectan muchos defectos técnicos y formales en los pliegos e incoherencias que perjudican a la libre competencia entre empresas y retrasa mucho los procesos al impugnarse los concursos.

\section{9.-Referencias}

[1] Gago-Calderón, A., \& Fraile, J. (2012). Iluminación con tecnología LED. Editorial Paraninfo.

[2] Valdecantos, A. B. (2015). Calidad y seguridad de productos de iluminación. Economía industrial, (396), 121-130.

[3] IDAE \& CEI. (2015). Requerimientos Técnicos Exigibles para Luminarias con Tecnología LED de Alumbrado Exterior. Rev. 4-120815 http://www.idae.es/uploads/documentos/documentos Requerimientos LED RE V-4-120815_81a949fd.pdf Visto por última vez el 09/08/2016

[4] Buchiniz, Y., Torre, G., \& Lépez, H. (2010). El valor del recurso cielo-paisaje nocturno y la contaminación lumínica. In Actas del VI Congreso Nacional Ambiental.

[5] Barba, M. N. Coordinador (2008). Grupo de trabajo: Contaminación Lumínica. Congreso Nacional del Medio Ambiente 9. CONAMA. Diciembre 2008 http://www.celfosc.org/biblio/general/conama92008.pdf Visto por última vez el 09/08/2016 
WPSReview International on Sustainable Housing and Urban Renewal

(RI-SHUR)

[6] Orduña, A. d. (2016). Pliego de cláusulas administrativas particulares para la contratación de la fase II del suministro e instalación de la renovación del alumbrado exterior municipal de Orduña (Bizkaia), por procedimiento abierto, varios criterios, tramitación ordinaria. Orduña.

http://w w w.urduna.com/es-E S/Ayuntami ento/Perfi I -C ontr atante/Pagi nas/ Tr ami taci on_Li ci taci \%C3\%B3ndel contr atodesumi ni stroei nstal aci \%C3\% B3ndelarenovaci\%C3\%B3ndelalumbradoexteriormunicipalFasell.aspx Visto por última vez el 09/08/2016

[7] Andalucía, J. d. (2016). Resolución del Tribunal Administrativo de Recursos Contractuales de la Junta de Andalucía. Recurso 242/2015. Sevilla

http://www.juntadeandalucia.es/export/drupaljda/7 16 RCT 24215 E Ayunta miento\%20 Dos\%20Hermanas\%20-\%20GAMMA\%20P.pdf Visto por última vez el 09/08/2016 